\title{
Steroidprämedikation: Wie lässt sich die Adhärenz optimieren?
}

\begin{abstract}
Die prophylaktische Gabe eines Steroids kann die Inzidenz von Überempfindlichkeitsreaktionen und Flüssigkeitsretention unter einer Therapie mit Docetaxel deutlich reduzieren. Allerdings ist die Adhärenz bezüglich der Steroidanwendung suboptimal. Daher wurden Onkologen, Apotheker, Krankenschwestern und Brustkrebspatientinnen über ihre Erfahrungen mit der Steroidprämedikation vor der Anwendung von Docetaxel befragt.

$53,8 \%$ der eingeladenen Krankenschwestern, 19,7\% der Apotheker und $14,8 \%$ der Onkologen beantworteten die Fragebögen. Es zeigte sich, dass zwei Steroidschemata am häufigsten zur Anwendung kamen: Dexamethason 8 mg zweimal täglich, wobei entweder sechs (79\%) oder zwei (11\%) Dosen vor der Docetaxel-Anwendung verabreicht wurden. Nur $2 \%$ der befragten Institutionen verordne-
\end{abstract}

\section{kurz notiert}

Forschungsstandort Deutschland

Interdisziplinäre Lungenkrebstherapie +++ Soll man bei fortgeschrittenem, aber nicht metastasiertem Lungenkarzinom eine hochdosierte Radiochemotherapie durchführen oder eine risikoadaptierte Chemotherapie und zusätzlich eine Operation? Dieser Frage gingen Essener Wissenschaftler in der Studie ESPATUE nach. Dabei zeigte sich, dass mit beiden Verfahren vergleichbare 5-Jahres-Gesamtüberlebensraten von 40 bzw. $44 \%$ erreicht wurden. Die Therapieentscheidungen sollten daher in interdisziplinärer Zusammenarbeit getroffen werden, je nach individueller Risikokonstellation eines Patienten, so Studienleiter Wilfried Eberhardt [Eberhardt WEE et al. J Clin Oncol. 2015;33(35):4194-201]. red.

Neue Strategie bei Pankreaskarzinom +++ Die Menge der T-Zellen, in der ein alternativer p38-Signalweg genutz wird, ist mit der Aggressivität eines Pankreaskarzinoms und mit der Menge an CD4+-Lymphozyten assoziiert, die proinflammatorische und tumorfördernde Zytokine produzieren. Heidelberger Forscher konnten nun in Tierversuchen zeigen, dass das Krebswachstum durch die medikamentöse Inhibition dieses alternativen Signalwegs gebremst werden kann [Alam S et al. Nat Med. 2015;21(11):1337-43]. ten lediglich eine Dexamethason-Dosis. Die Adhärenz gegenüber der Steroidprämedikation war bei den meisten Patienten suboptimal, wie $98 \%$ der befragten Ärzte, Apotheker und Schwestern angaben. Die Patienten ließen einmal pro Tag (3\%), einmal pro Woche (33\%), einmal pro Monat (26\%) oder einige Male im Jahr (27\%) eine Dosis aus.

In $65 \%$ der Institutionen liegen lokale Protokolle zum Management von Patienten vor, die ihre Prämedikation nicht korrekt anwenden. Allerdings kamen in den verschiedenen Einrichtungen unterschiedliche Strategien zur Anwendung.

Die Erfahrungen von Ärzten, Apothekern und Pflegekräften stimmten nicht mit den Angaben der Patientinnen überein. Die Patientenfragebögen wurden von 82,3\% der Eingeladenen ausgefüllt. 99\% der befragten Patientinnen gaben an,
Die Gabe eines Steroids vor der Therapie mit Docetaxel soll Nebenwirkungen reduzieren.

dass sie ihre Prämedikation korrekt eingenommen haben. Die Patientinnen führten außerdem an, dass Steroide häufig Nebenwirkungen verursachen würden, vor allem Schlafstörungen (49\%), Hautreaktionen (22\%), Hunger und Gewichtszunahme (17\%). Darüber hinaus gaben $46 \%$ der Patientinnen an, dass ihnen schriftliche Hinweise die regelmäßige Einnahme der Medikation leichter machen könnten.

Judith Neumaier

Jacobs C et al. Optimisation of steroid prophylaxis schedules in breast cancer patients receiving docetaxel chemotherapy - a survey of health care providers and patients. Support Care Cancer. 2015;23(11):3269-75.

BRAF-mutiertes Melanom

\section{Erhöhte Lebensqualität unter der Therapie mit BRAF- plus MEK-Inhibitor}

Bei Patienten mit metastasiertem Melanom und nachgewiesener BRAF-V600Mutation bietet die Kombination aus BRAF- plus MEK-Inhibitor gegenüber einer BRAF-Inhibitor-Monotherapie nicht nur einen signifikanten Vorteil beim Gesamtüberleben, sondern geht auch mit einer verbesserten Lebensqualität einher.

In der offenen Phase-III-Studie COMBI-v mit 704 therapienaiven Patienten mit metastasiertem Melanom und einer BRAF-V600-Mutation wurde die Kombination aus dem BRAF-Inhibitor Dabrafenib und dem MEK-Inhibitor Trametinib mit einer Monotherapie mit dem BRAF-Inhibitor Vemurafenib verglichen. Dabei zeigte sich ein deutlicher Überlebensvorteil zugunsten der Kombination. Die Studie wurde vorzeitig gestoppt und den Patienten der Monotherapiegruppe ein Wechsel in die Kombinationsgruppe angeboten.
$\mathrm{Zu}$ Beginn der Behandlung waren die Angaben in den gesundheitsbezogenen Fragebögen zur Lebensqualität (HRQoL) vergleichbar. Im Verlauf der Studie verbesserten sich dann die Scores über nahezu alle Domänen unter der Kombination kontinuierlich und waren im Vergleich zur Monotherapie signifikant überlegen.

Auch diese Ergebnisse sprächen für die Kombinationstherapie, so die Autoren. Allerdings war dieser Studienendpunkt nicht mit genügend statistischer Power ausgestattet, sodass dieses Ergebnis noch bestätigt werden sollte. Barbara Kreutzkramp

Grob JJ et al. Comparison of dabrafenib and trametinib combination therapy with vemurafenib monotherapy on health-related quality of life in patients with unresectable or metastacic cutaneous BRAF Val600-mutation-positive melanoma (COMBI-v): results of a phase 3, open-label, randomised trial. Lancet Oncol. 2015;16(13):1389-98. 